\title{
MASS-RATIO DETERMINATION IN CONTACT BINARIES
}

\author{
J. B. HUTCHINGS \\ Dominion Astrophysical Observatory, Victoria, B.C., Canada
}

\begin{abstract}
In the wake of recent theoretical work on contact systems (e.g. Whelan, 1972; Biermann and Thomas, 1972; Lucy, 1968), it is of importance to determine fundamental data from observations. This has been done recently by several groups in analysing light curves (Mochnacki and Doughty, 1972; Hutchings and Hill, 1973; Wilson and Devinney, 1973), and it is found that shapes, temperature differences and distributions, and mass-ratios, can be determined in many cases. However, where spectroscopic data are also available, the mass-ratios are not always in agreement. Using the photometric models, it is possible (Hutchings, 1973) to calculate the distortion of line profiles resulting (primarily) from the non-uniform brightness over the component stars in these systems. This distortion leads to the characteristically observed 'square' velocity curves for the systems (e.g. Binnendijk, 1967). Correction for the effect in most cases (a) resolves the mass-ratio discrepancy and (b) leads to better estimates for the masses. The faintness of most contact systems makes detailed spectroscopy difficult, but there appears to be a need for further work in the directions outlined here to improve the fundamental data available on them. These results should also be borne in mind in inspecting previous work on contact binaries.
\end{abstract}

\section{References}

Biermann, P. and Thomas, H. C.: 1972, Astron. Astrophys. 16, 60.

Binnendijk, L.: 1967, Publ. Dominion Astrophys. Obs. 13, 27.

Hutchings, J. B. : 1973, Astrophys. J. 180, 501.

Hutchings, J. B. and Hill, G. : 1973, Astrophys. J. 179, 539.

Lucy, L. B. : 1968, Astrophys. J. 153, 877.

Mochnacki, S. and Doughty, N. A.: 1972, Monthly Notices Roy. Astron. Soc. 156, 243.

Whelan, J. A. J.: 1972, Monthly Notices Roy. Astron. Soc. 156, 115.

Wilson, R. E. and Devinney, E. J.: 1973, Astrophys. J. 182, 539. 